
Not for reproduction, distribution or commercial use.

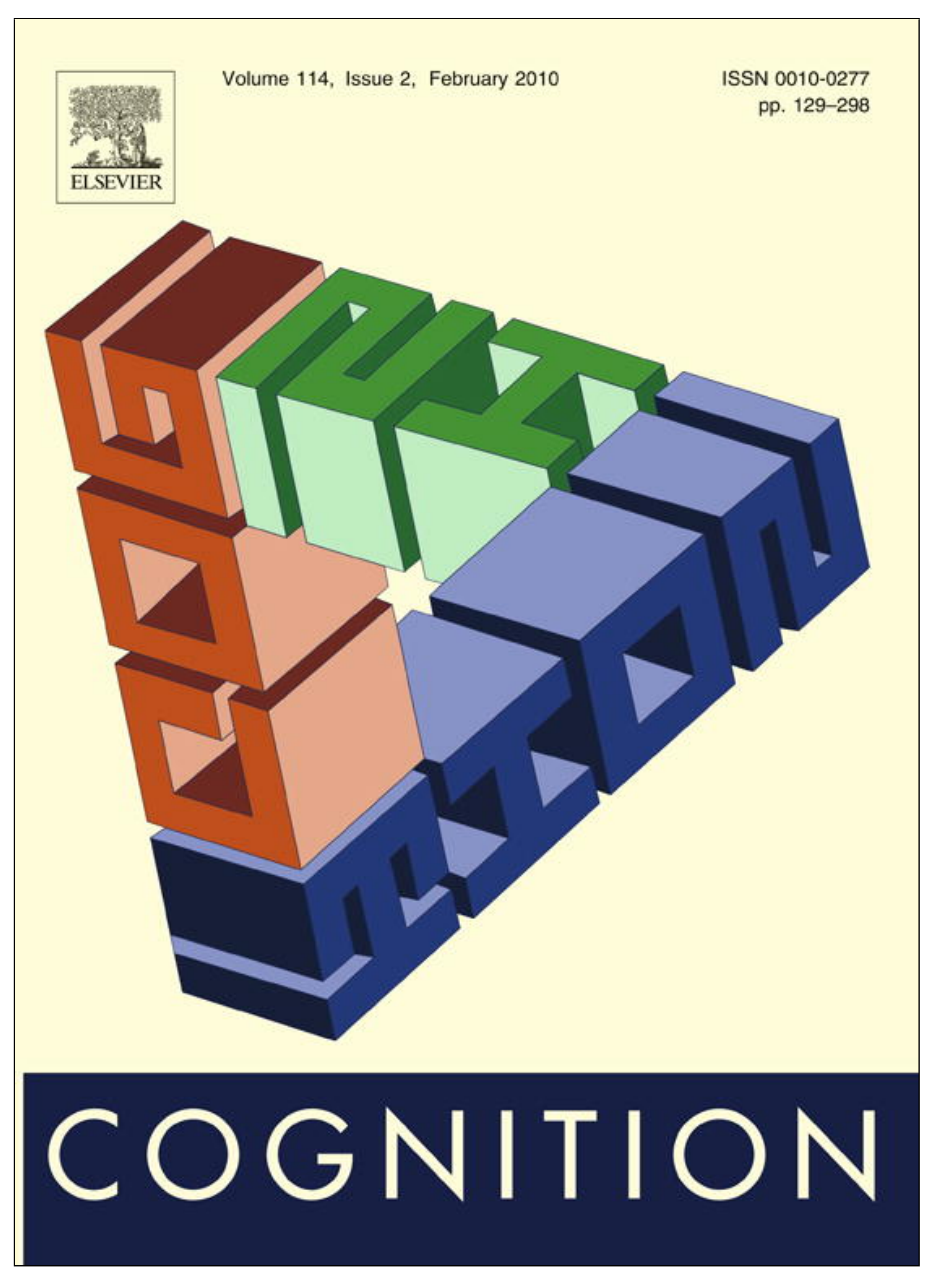

This article appeared in a journal published by Elsevier. The attached copy is furnished to the author for internal non-commercial research and education use, including for instruction at the authors institution and sharing with colleagues.

Other uses, including reproduction and distribution, or selling or licensing copies, or posting to personal, institutional or third party websites are prohibited.

In most cases authors are permitted to post their version of the article (e.g. in Word or Tex form) to their personal website or institutional repository. Authors requiring further information regarding Elsevier's archiving and manuscript policies are encouraged to visit:

http://www.elsevier.com/copyright 


\title{
Infants' understanding of everyday social interactions: A dual process account
}

\author{
Gustaf Gredebäck*, Annika Melinder \\ The Cognitive Developmental Research Unit (EKUP), Department of Psychology, University of Oslo, Norway
}

\section{A R T I C L E I N F O}

\section{Article history:}

Received 4 March 2008

Revised 2 September 2009

Accepted 6 September 2009

\section{Keywords:}

Action understanding

Learning

Direct matching

Eye tracking

Experience

Feeding anticipation

Teleological stance

Mirror neurons

\begin{abstract}
A B S T R A C T
Six- and 12-month-old infant's eye movements were recorded as they observed feeding actions being performed in a rational or non-rational manner. Twelve-month-olds fixated the goal of these actions before the food arrived (anticipation); the latency of these gaze shifts being dependent $(r=.69)$ on infants life experience being feed. In addition, 6- and 12-month-olds dilated their pupil during observation of non-rational feeding actions. This effect could not be attributed to light differences or differences in familiarity, but was interpreted to reflect sympathetic-like activity and arousal caused by a violation of infant's expectations about rationality. We argue that evaluation of rationality requires less experience than anticipations of action goals, suggesting a dual process account of preverbal infants' everyday action understanding.
\end{abstract}

(c) 2009 Elsevier B.V. All rights reserved.

\section{Introduction}

Infants demonstrate a remarkable range of social skills that help them understand the actions and intentions of others. With this understanding infants start making sense of, and take part in, the complex social interactions that define human interpersonal exchange. In fact, as soon as infants become proficient at imitating complex actions they already possess the ability to distinguish rational from non-rational actions and imitate action goals rather than observed movements (Carpenter, Akhtar, \& Tomasello, 1998; Gergely, Bekkering, \& Király, 2002; Meltzoff, 1995).

However, the foundation of action understanding emerges much earlier. Resent studies demonstrate that infants from 6.5 months of age retrospectively evaluate the interaction between a wide range of animated and human characters as rational or non-rational (Csibra, 2008; Csibra, Bíró, Koós, \& Gergely, 2003; Kamewari, Kato, Kanda, Ishiguro, \& Hiraki, 2005; Wagner \& Carey, 2005). These studies

\footnotetext{
* Corresponding author.

E-mail address: gustaf.gredeback@psykologi.uio.no (G. Gredebäck).
}

all presented infants with self-propelled agents that move passed an obstacle to reach a goal (rational movement). Once habituated, infants were presented with similar events in which the obstacle had been removed. In these test trials infants appeared surprised if the agent continued on the old, non-rational path but not if the agent changed to a new and, given the current context, rational path to reach the goal. In this context it is important to note that these studies investigate how infants' overall looking time changes over successive action sequences. Infants' reactions are, as such, based on evaluations of past event.

Given that infants evaluate the rationality of a wide range of self-propelled agents it is not likely that this evaluation is solely based on infants own experience with similar events (Csibra, 2008). In fact, it has been proposed that infants' evaluations of rationality are dependent on abstract well-formedness principles that are applicable to a wide range of stimuli (see the teleological stance, Gergely \& Csibra, 2003; Király, Jovanovic, Prinz, Aschersleben, \& Gergely, 2003). These processes are assumed to help infants interpret the world, independent of their own experience with similar events. According to Csibra et al. 
(2003) teleological reasoning might even be applicable to ongoing events, allowing infants to anticipate the goal of others actions independent of prior experience. However, the involvement of teleological reasoning in goal anticipation is currently under debate (Eshuis, Coventry, \& Vulchanova, 2009; Falck-Ytter, Gredebäck, \& von Hofsten, 2006).

In fact, even though infants have the ability to evaluate the rationality of numerous self-propelled agents independently of prior experience with similar events, their ability to anticipate the goal during observation of object manipulations (here defined as the ability to fixate the goal of an action before it is accomplished) appears specifically structured around human actions that infants have experience performing. Recently, Falck-Ytter et al. (2006) measured 6-, 12-month-olds, and adults gaze as they observed an adult placing three toys in a bucket. Both 12-month-olds and adults fixated the bucket before the action was completed, thereby anticipating the action goal. However, when the toys moved on their own both groups tracked the toys reactively. Six-month-olds tracked both human actions and animated toys in a reactive manner. This result suggests that infant's ability to anticipate the goal of ongoing actions is closely associated with human actions, and in particular, actions that infants can perform by themselves. The later statement is supported by the observation that infants become proficient at placing objects inside buckets between 6 and 12 months of age (Bruner, 1970), the same age in which infants develops the ability to anticipate the goal of such actions (Falck-Ytter et al., 2006).

These findings receive support from several studies that investigate infants' evaluations of past events, all of which focus on the ability to encode the goal of others actions (Sommerville, Woodward, \& Needham, 2005; Woodward, 1998). Unlike goal anticipation, the ability to encode the goal of observed actions is not limited to human actions (Johnson, Shimitzu, \& Ok, 2007; Luo \& Baillargeon, 2005; Shimizu \& Johnson, 2004). However, infant's ability to encode the goal of observed events are strengthened if the observed events match ones own experiences and manual capabilities. For example, Woodward (1998) presented infants with a human actor who reached for one of two toys. After habitation the toys changed place and the actor either reached for the same toy in a new location or a new toy at the old location. Five-, 6-, and 9-month-old infants looked longer when the actor reached for the new toy but not when the actor reached along a new path to reach the old toy. This selective attention to the goal of human reaching actions did not transfer to similar actions performed by a mechanical claw. Similar results were demonstrated at 3 months of age, if infants were trained on a reaching task prior to habituation (Sommerville et al., 2005).

This link between infants' own action capabilities and their understanding of others actions has recently received much attention (Csibra \& Gergely, 2007; Meltzoff, 2007). It has been proposed that action production and action observation are integrated through a direct matching process (Flanagan \& Johansson, 2003) that encode both context specific actions and the goals of those actions (see the mirror neuron system, Iacoboni \& Dapretto, 2006; Rizzolatti \& Craighero, 2004).

Based on these results it is tempting to assume that infants' evaluations of rationality are less dependent on experience than infants' ability to encode and anticipate the goal of manual actions. However, no studies have explicitly attended to how infant's everyday experience modulates their understanding of others actions. One reason might be that studies of developmental social cognition, and particularly studies of action understanding, present infants with relatively sparse and uniform events that are far removed from the complex social interactions that infant face on an everyday basis. In fact, most of the social encounters that we partake in, or observe, involve a succession of human actions performed on different objects (Land \& Hayhoe, 2001) and towards different individuals.

The current paper attempts to, in an ecologically relevant context, record 6- and 12-month-olds gaze as they observe two adults how converse and perform a sequence of everyday actions (some of which are rational and others that are non-rational). Numerous studies have relied on eye tracking and anticipation to assess both representational abilities (Gredebäck \& Von Hofsten, 2007) and action understanding (Falck-Ytter et al., 2006) in infancy. The measure warrants, as such, little additional introduction. In addition, we rely on changes in pupil size to assess infant's abilities to retrospectively evaluate the rationality of observed events; this measure is novel to the infancy literature and has primarily been used to assess emotional and cognitive processing in adults (Libby, Lacey, \& Lacey, 1973; Tryon, 1975; Võ et al., 2007). In this literature, changes in pupil size are thought to reflect changes in sympathetic-like activity (Libby et al., 1973), arousal, and cognitive load during information processing (Bradshaw, 1967). More specifically, pupil dilation has been observed following presentation of interesting, attention grabbing and unusual events (Libby et al., 1973). Our design allows us to, simultaneously, map out infants abilities to anticipate the goal of ongoing actions and their ability to evaluate the rationality of observed action goals; relating both of these markers of action understanding to infant's everyday experience with similar events.

\section{Experiment 1}

\subsection{Methods}

\subsubsection{Participants}

Fifty-six infants (26 female) were randomly assigned to either the Rational or Non-Rational condition. The mean age equaled 6 months, 8 days $(S D=11)$ and 12 months, 6 days ( $S D=8$ days $)$ in the Rational condition vs. 6 months, 2 days ( $S D=10$ days) and 12 months, 5 days ( $S D=5$ days $)$ in the Non-Rational condition. An additional six infants were excluded due to fussiness or calibration failure.

\subsubsection{Stimuli and apparatus}

Gaze and pupil size was measured using a Tobii 1750 near infrared eye tracker with an infant add-on; precision 
$1^{\circ}$, accuracy $0.5^{\circ}$, and sampling rate $50 \mathrm{~Hz}$. A standard 9 point infant calibration was used (Falck-Ytter et al., 2006). The infants were presented with videos $(24.8 \times 20.7$ visual degrees $)$ of two adult females, referred to as feeder and recipient based on their respective tasks during feeding, engaged in a dyadic social interaction (Fig. 1). Each video started with a conversation about the fact that both were hungry, followed by eating actions, and thereafter, feeding actions. During eating actions, the feeder placed a plate of bananas in front of her, reached for a spoon and ate three piece of banana, in succession. Feeding actions started with the recipient asking for a taste. The feeder agreed and scooped up a piece of banana, holding the spoon stationary as the recipient opened her mouth and simultaneously moved her hand sideways. Once this movement was completed the feeder said 'here it comes', moving the spoon towards the recipient. The feeding action was repeated three times. In the Rational condition the feeder moved the spoon to the recipient's mouth. In the Non-Rational condition the feeder placed the banana on the back of the recipient's hand, after which, the recipient leaned forward and ate from the back of her hand.

The video ended after a brief conversation about how tasty the banana was and once the feeder had removed the plate. With one exception, only one movement or conversation phrase occurred at a time. However, during feeding the recipient opened her mouth and moved her hand simultaneously. The later was necessary to ensure an equivalent degree of movement in both potential goal areas prior to the feeding action.

\subsubsection{Procedure}

The study was approved by the Regional Ethic Committee according to the 1964 Declaration of Helsinki. Participants were recruited by mail. As each family entered the lab parents were informed about the procedure, signed a consent form, and asked about their infant's experience eating, being feed, and feeding others. Following these questions, infants were seated in a safety car seat on the parent's lap and performed a calibration procedure. During the experimental session infants were presented with six videos of either the Rational or Non-Rational condition, interleaved with brief animations designed to orient their attention to the screen. Each family spent approximately $20 \mathrm{~min}$ in the lab and was given a small gift (12€).

\subsubsection{Data reduction}

Initial data reduction was performed in Adobe Premiere with a frame by frame analysis of Gaze Reply movies from ClearView (Tobii). These movies included both the location of infants gaze and the video that infants watched (time locked, $50 \mathrm{~Hz}$ ). To account for spatial sampling errors a 0.8 visual degree outline was added outside the plate as well as both agent's heads and hands (AOI's). Future reference to the location of infants fixations refer to these AOI's. Statistical analyses were performed using General Linear Models (GLM) and Sheffe's post hoc tests $(p<.05)$.

Feeding events were included in the analysis if infants shifted their gaze from the feeder to the recipient during the feeding action. More specifically, infants had to fixate the plate, hand, or head of the feeder after she started saying 'here it comes', but before the spoon passed the middle of the table. In addition, infants had to shift their gaze to the recipient, fixating her head or hand, before the spoon was retrieved by the feeder. A fixation was defined as a stable gaze (within 0.8 visual degrees) for at least $200 \mathrm{~ms}$ (Engel, Anderson, \& Soechting, 1999; Gredebäck, Örnkloo, \& von Hofsten, 2006).

Latency was calculated by subtracting the time when the spoon entered the AOI surrounding the head or hand from the onset of infant's first fixation within either of
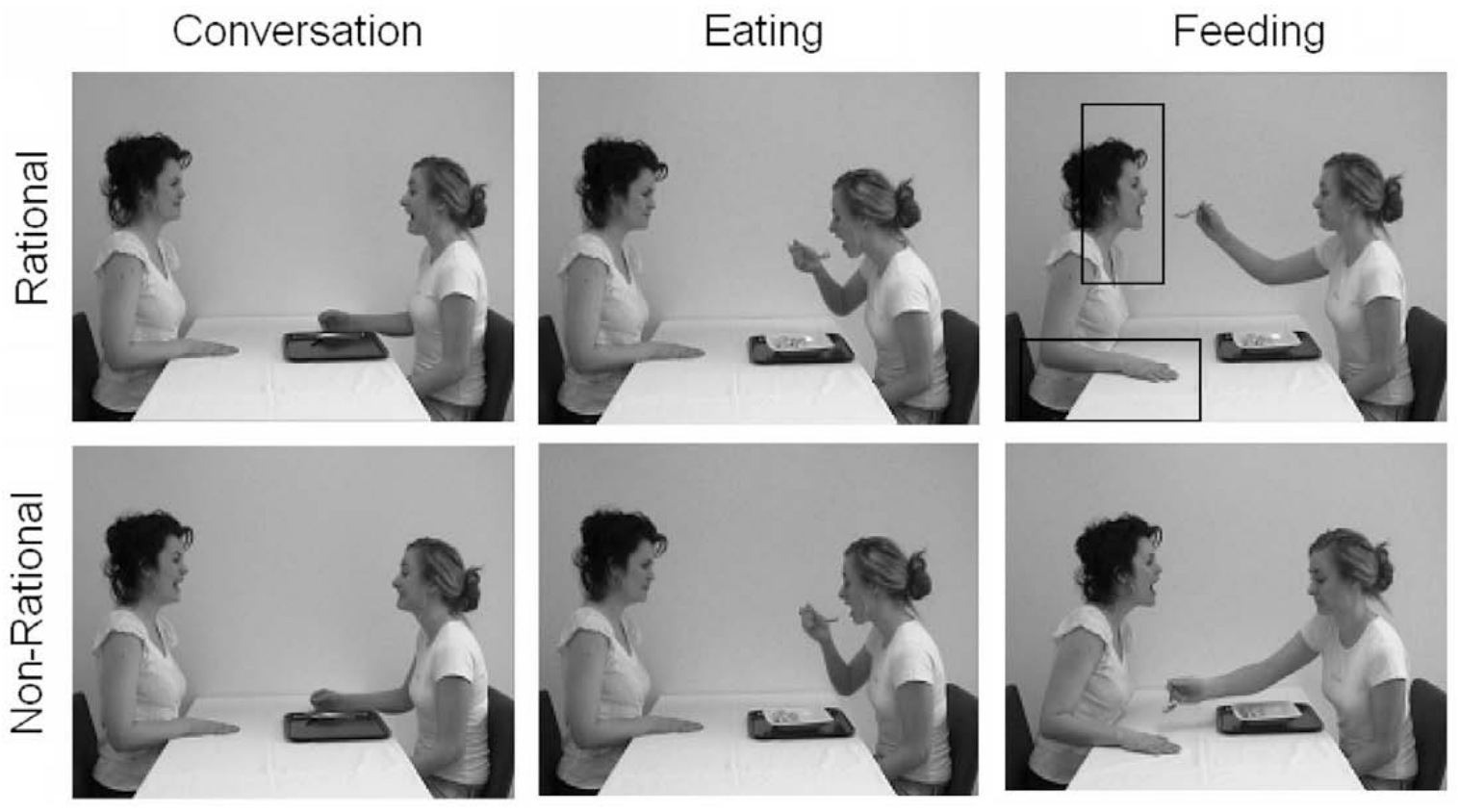

Fig. 1. Snapshots of the stimuli used in the Rational and Non-Rational condition of Experiment 1. Goal areas are marked with black rectangles on the picture depicting the feeding action in the Rational condition. 
these AOI's. Providing a measure of whether infants anticipated (negative latencies) the observed actions or if they tracked observed events in a reactive manner. The location of this fixation (at the head or hand) was also recorded. Preliminary analysis of latency and location did not find any differences between the six movies of each session; data were therefore collapsed across movies in the location analysis. In each of the above mentioned analysis the dependent measure was entered into a 2 (condition; rational vs. non-rational) $\times 2$ (age; 6 vs. 12 months) $\times 3$ (action; first vs. second vs. third feeding action) ANOVA with the last factor repeated.

\subsubsection{Pupil size}

For included feeding events the average pupil size was calculated in two 4-second blocks (horizontal error bars above Fig. $3 \mathrm{~A}$ and $\mathrm{B}$ ). The first block started following the arrival of the spoon at the AOI covering the head (Rational condition) or hand (Non-Rational condition) and lasted until the spoon had been removed and halfway through the chewing. The second block covered remaining chewing and swallowing, ending right before the recipient asked for more. Change in pupil size was calculated by subtracting the first block from the second block. Data was aggregated to a single data point per subject and entered into a 2 (condition; rational vs. non rational) $\times 2$ (age; 6 vs. 12 months) ANOVA. The frequency distribution of pupil dilation and contraction was also tested against random performance using non-parametric One-Way Goodness of Fit $\chi^{2}$.

An experience analysis was also performed, measuring the relationship between infants current (average no. of events/day in the last week) and life time (days since onset) experience being feed, eating, and feeding others with data from the latency and pupil size analysis. Statistical analysis of latency was performed using linear regression with experience as the independent variable. All parents reported that the rational feeding action, but not the non-rational feeding actions, reflected the infants' normal feeding pattern.

\subsection{Results and discussion}

\subsubsection{Latency}

As seen in Fig. 2, 12-month-olds fixated the recipient of the food before the spoon arrived, whereas 6-month-olds tracked the spoon reactively, $F(1,46)=28.2, p<.00001$, $\eta_{p}^{2}=.38$. The latency to fixate the recipient decreased (faster anticipations) over successive feeding actions, $F(2,92)=6.46, p<.003, \eta_{p}^{2}=.12$. Post-hoc tests demonstrated lower latencies during the third feeding action than during either of the first two feeding actions $(p<.05)$. This change over feeding actions was most pronounced in the Rational condition at 12 months of age, as demonstrated by a three way interaction between feeding action, condition, and age, $F(2,92)=4.13, p<.02, \eta_{p}^{2}=.08$. Post-hoc tests differentiated the third feeding action of the Rational condition at 12 months from all three actions in both condition at 6 months of age $(p<.05)$. There was also a two way interaction between feeding action and condition, $F(2,92)=4.1, p<.02, \eta_{p}^{2}=.08$. Post-hoc tests differentiate the third feeding action in the Rational condition from feeding actions one and two of the Rational condition as well as feeding action one of the Non-Rational condition

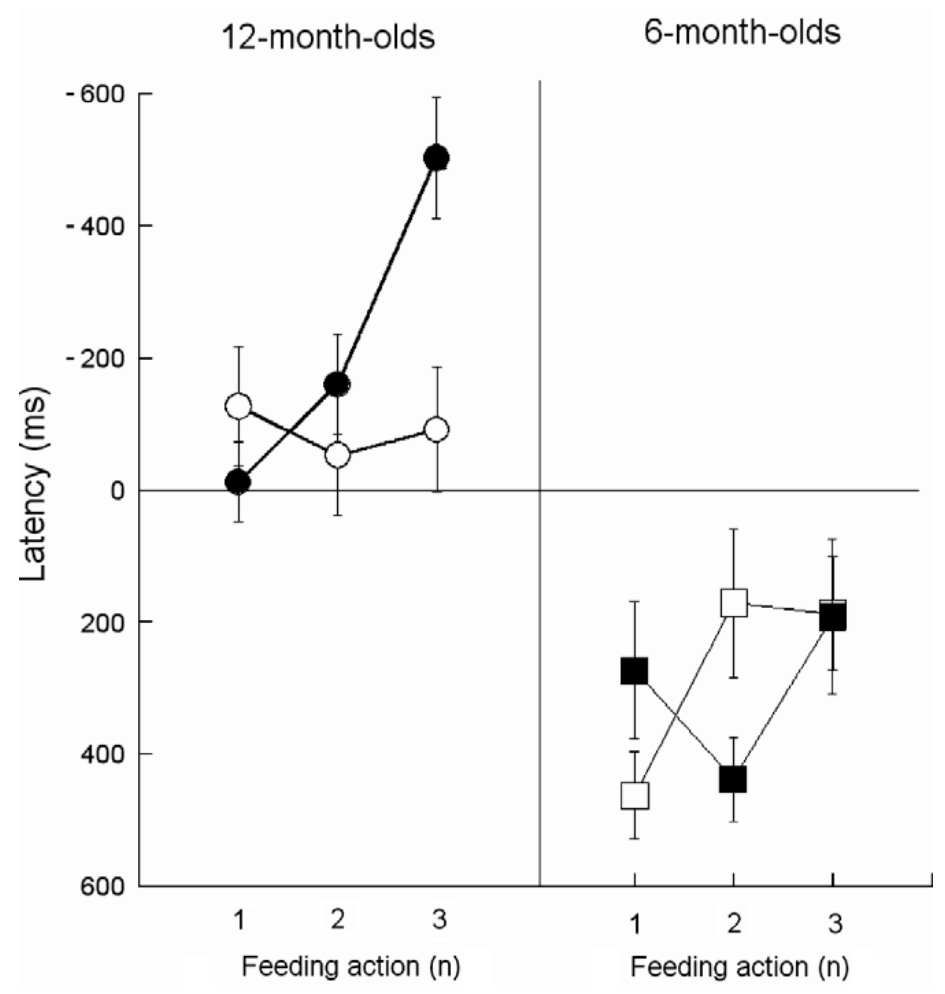

Fig. 2. Average latency to fixate the recipient (head or hand) in the Rational (filled) and Non-Rational (open) conditions, separate for the three feeding actions and the two age groups; 6- (squares) and 12-month-olds (circles). Error bars represent SE. 

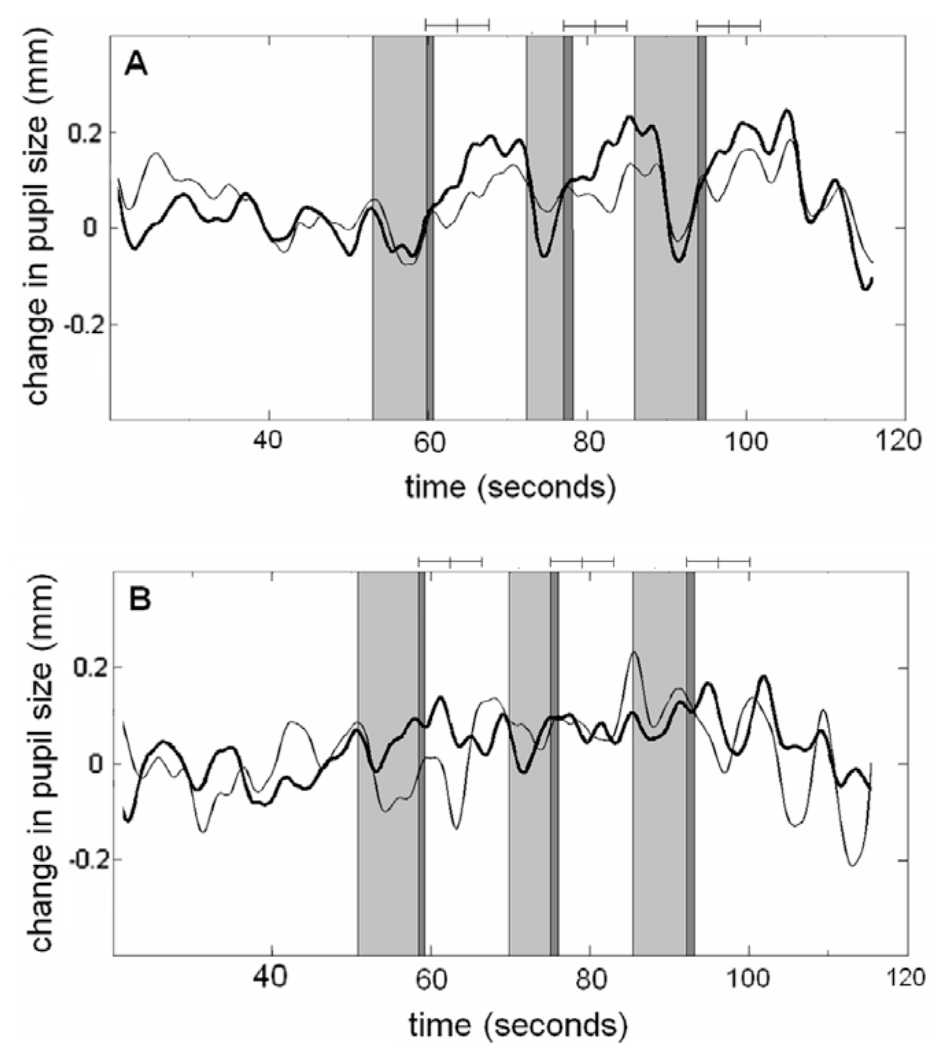

Fig. 3. Change in pupil size in the Non-Rational (A) and Rational (B) condition. Separate for 12- (thick line) and 6-month-olds (thin line). The light grey area represents the movement of the spoon towards the recipient and the dark grey area represents the time when the spoon is in contact with the recipient. Horizontal error bars on top of each figure represents the two blocks used in the analysis. Both areas and error bars are presented separately for each of the three actions of each video.

$(p<.05)$. In summary, the latency analysis demonstrate reactive gaze shifts in both conditions at 6 months of age, anticipatory gaze shifts in both conditions at 12 months of age, and enhanced anticipations during the last feeding action of the Rational condition at 12 months of age.

\subsubsection{Location}

Anticipations were directed towards the recipients face in both conditions and age groups (see Table 1). Reactive gaze shifts, on the other hand, were directed towards the recipients face in the Rational condition and towards her hand in the Non-Rational condition. This is expressed as an interaction between the anticipatory vs. reactive gaze shifts and condition, $F(1,39)=92.9, p<.00001, \eta_{p}^{2}=.70$, and validated by subsequent post-hoc tests $(p<.05)$. There was also a significant main effect of condition, $F(1,39)=61.9, p<.00001, \eta_{p}^{2}=.61$, and a significant difference between where infants looked during anticipatory

Table 1

Percentage of gaze shifts directed towards recipients head, separate for anticipations and reactions in each of the two conditions; $100^{*}(n$ predictions to head $/(n$ predictions to head $+n$ predictions to hand $)$ ). Italic numbers denote cells where gaze was primarily directed towards the hand.

\begin{tabular}{llrlll}
\hline & \multicolumn{2}{l}{ Rational } & & & \multicolumn{2}{l}{ Non-Rational } \\
\cline { 2 - 3 } \cline { 5 - 6 } & 6 & & & 6 & 12 \\
\hline Anticipations & 100 & 78 & & 86 & 94 \\
Reactions & 100 & 100 & & 16 & 25 \\
\hline
\end{tabular}

and reactive gaze shifts, $F(1,39)=51.4, \quad p<.00001$, $\eta_{p}^{2}=.57$. No significant age effects were observed. These results demonstrate that infant's anticipations were directed to the mouth in both conditions, whereas reactive gaze shifts were directed to the mouth in the Rational condition and to the hand in the Non-Rational condition.

\subsubsection{Pupil size}

Pupil size was measured continuously and aggregated in two 4 second blocks covering the placement of the food and the subsequent eating. In the Non-Rational condition, $79 \%$ of the 12-month-olds (One-Way Goodness of Fit $\left.\chi^{2}(1)=10.3, p<.05\right)$ and 93\% of the 6-month-olds (OneWay Goodness of Fit $\chi^{2}(1)=4.6, p<.05$ ) dilated their pupils from the first to the second block. Contrary, in the Rational condition, $86 \%$ of the 12-month-olds (One-Way Goodness of Fit $\left.\chi^{2}(1)=7.14, p<.05\right)$ and $57 \%$ of the 6 -month-olds (n.s.) contracted their pupil. The change in pupil size differed significantly between conditions, $F(1,51)=23.4$, $p<.0001, \eta_{p}^{2}=.31$. No differences were observed between the two age groups.

\subsubsection{The role of experience}

A detailed description of infants feeding experience can be found in Table 2; together with information about infants' eating experience and their experience trying to feed others. No difference in experience was observed between the Rational and Non-Rational condition, as such, descriptive statistics of experience are aggregated across 
Table 2

Infant's life time experience and daily experience (within last week) performing the observed actions. Numbers in parenthesis represent $S D$ and range. Note. Latency during feeding actions did not correlate with infants experience eating or feeding others, even though the range (in days) is equivalent between the three experience measures at 12 months of age (see table experience).

\begin{tabular}{|c|c|c|c|c|}
\hline & \multicolumn{2}{|l|}{6} & \multicolumn{2}{|l|}{12} \\
\hline & Life (months) & Daily (times) & Life (months) & Daily (times) \\
\hline Being feed with spoon & $1.4(0.8,0-3)$ & $1.9(1.1,0-3.5)$ & $7.0(1.0,5-9)$ & $2.8(1.1,0.5-5)$ \\
\hline Feeding others & - & - & $1.0(1.0,0-3.5)$ & $1.2(1.2,0-5)$ \\
\hline
\end{tabular}

conditions. However, the relationship between experience and latency demonstrated clear differences between conditions. Twelve-month-old infants average latency to fixate the recipient during normal feeding actions (Rational condition) was dependent on their life experience being feed, $r=0.69 ; F(1,11)=10.07, p<.01, \eta_{p}^{2}=.48$ (Fig. 4); demonstrating that infants with much experience fixated the recipient earlier than infants with little experience. None of the other experience measures predicted the latency of infants' goal fixation or changes in pupil size. Infants experience did not influence the latency of infants gaze shifts when observing the Non-Rational condition.

Experiment 1 demonstrates that the latency of 12month-old infants goal anticipations were dependent on the infants own experience with similar action sequences. In addition, both 6- and 12-month-olds retrospective evaluations of rationality (as measured by pupil size) demonstrate a higher degree of experience independence. Both age groups dilate their pupil more during observation of rational actions than during observation of action sequences that are non-rational. Unfortunately, Experiment
1 is not able to disentangle whether these differences are related to the rationality of the action or to differences in familiarity or novelty of the actions being observed; the rational action also being familiar (depicting normal feeding) at the same time as the non-rational action is unfamiliar. It is perceivable that infants respond with increased pupil dilation to unfamiliar rather then non-rational social events. Experiment 2 aims to disentangle these alternative accounts of infants pupil dilation.

\section{Experiment 2}

Experiment 2 presents a group of 6-month-olds with two feeding conditions in which the feeder place pieces of banana on the back of the recipients hand. In one condition a barrier blocks the feeders' access to the recipients mouth whereas the other conditions includes a barrier that does not limit the two actors action space. Experiment 2 focuses on 6-month-olds for several reasons. First, only a few studies have demonstrate action understanding capabilities during the infants first 6 months of life

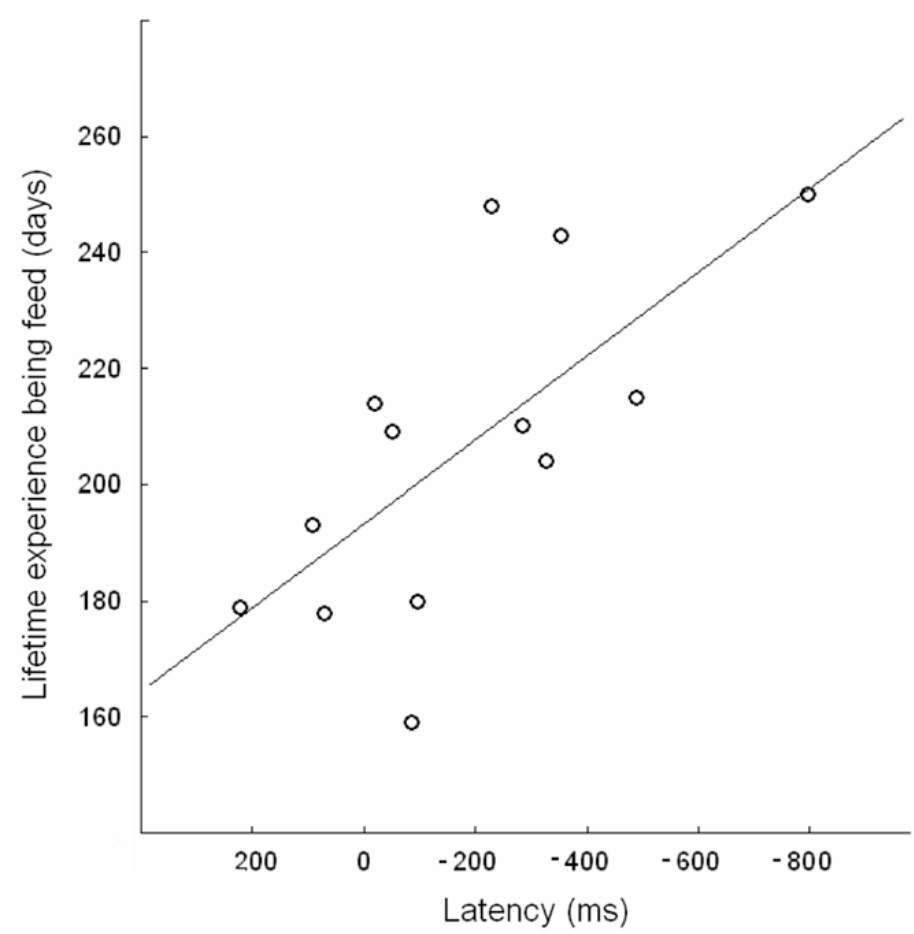

Fig. 4. Correlation $\left(r_{\mathrm{xy}}=.69\right)$ between lifetime experience (in days) being feed and the latency to fixate the recipient during observation of feeding actions at 12 months of age in the Rational condition. Note. The regression line in figure correlation suggests that infants require 193 days of experience to anticipate normal feeding actions in this context. 


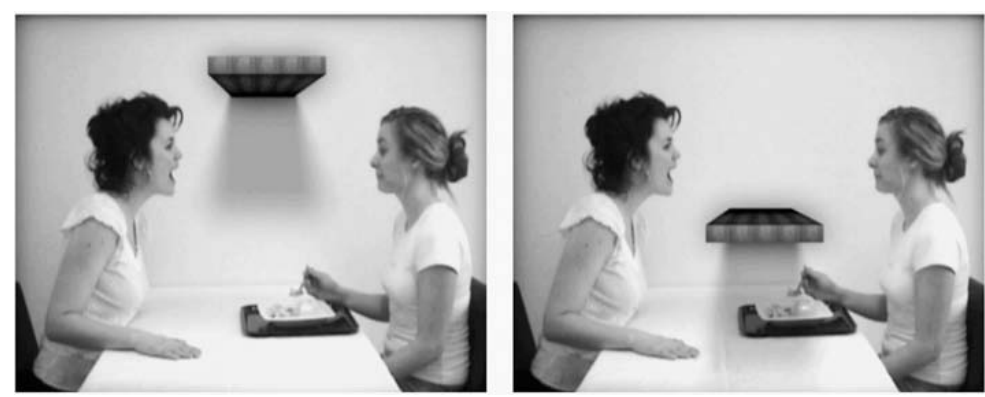

Fig. 5. Snapshots of the stimuli used in Experiment 2. Both conditions featured identical feeding actions directed towards the recipient's hand.

(see Section 1), making it critical to replicate this finding. Second, the close similarity between the pupil dilations of 6- and 12-month-olds in Experiment 1 allow us to focus our attention to the youngest age at which the effect manifests itself, enhancing our understanding of the processes that guide pupil dilation early in life.

\subsection{Methods}

\subsubsection{Participants}

Fourteen 6-month-old infants ( 7 female, mean age 6 months, 7 days, $S D=3$ days) participated in Experiment 2. All infants who participated were included in the final analysis.

\subsubsection{Stimuli and apparatus}

Participants were presented with reduced versions of the Non-Rational condition from Experiment 1; including only the feeding action that place food on the back of the recipients hand (initial conversation and eating actions were removed). Added to this video is a horizontal barrier that extends from the back wall (see Fig. 5). In the Non-Rational and Unfamiliar condition the barrier is placed outside the actors reaching space, as such, it does not restrict the feeder's access to the recipient's mouth. This action is regarded as non-rational because the feeder places the food on the back of the hand even though she can reach the recipients mouth. In the Rational and Unfamiliar condition the barrier is placed in between the feeder and the recipient; blocking the feeder's access to the recipient's mouth while at the same time maintaining free access to the recipient's hand. This action is regarded as rational because the feeder is only able to place the food on the back of the receiver's hand. Please note that the action performed in the two conditions is identical (and unfamiliar); only the location of the barrier differs. If the pupil size differences of Experiment 1 are caused by the rationality of the actions being observed we predict larger pupil dilations during the Non-Rational and Unfamiliar condition then during the Rational and Unfamiliar condition.

\subsubsection{Procedure}

The general procedure for recruitment and participation was identical to Experiment 1 with one exception. Each infant participated in both conditions on separate days. The order of the two conditions was counterbalanced across participants.

\subsubsection{Data reduction}

Pupil size was aggregated over two blocks. One block coved the movement of the spoon and subsequent contact between the spoon and the recipient's hand (total duration $2.4 \mathrm{~s}$ ) whereas the other block covered the $2.4 \mathrm{~s}$ prior to this movement ${ }^{1}$. Change in pupil size between these blocks was computed in the same manner as described in Experiment 1 . Preliminary analysis did not reveal any systematic effect of presentations order. As such, data was aggregated to two data points per subject (one for each condition) and compared across conditions using a one-way ANOVA (with condition as independent variable). The frequency distribution of change in pupil size over conditions was assessed using non-parametric One-Way Goodness of Fit $\chi^{2}$.

Experiment 2 was designed to evaluate the novel pupil dilations observed in Experiment 1. Anticipation data was not analyzed in Experiment 2 because the different locations of barriers in the two conditions made a direct comparison of infants scanning patterns (with respect to action understanding) difficult. From a methodological point of view, directly comparing the latency of infants' goal directed gaze shifts require a much higher degree of similarity between conditions (Gredebäck, Johnsson, \& von Hofsten, in press).

\subsection{Results and discussion}

\subsubsection{Pupil size}

In the Non-Rational and Unfamiliar condition $92 \%$ of infants dilated their pupil between the two blocks (One-Way Goodness of Fit $\chi^{2}(1)=9.31, p<.05$ ) whereas only $57 \%$ dilated their pupil between the two blocks in the Rational and Unfamiliar condition (n.s.). The change in pupil size differed significantly between conditions, $F(1,12)=16.87$, $p<.005, \eta_{p}^{2}=.58$ with more pupil dilation during observation of feeding actions in the Non-Rational and Unfamiliar condition (mean dilation $=.09 \mathrm{~mm}, S E=.01$ ) then during the Rational and Familiar condition (mean dilation $=.01$ $\mathrm{mm}, \mathrm{SE}=.02$ ).

\subsubsection{Experience}

Participating 6-month-olds did not eat by themselves, nor did they feed others. Some of the infants were, how-

\footnotetext{
1 Visual inspection of pupil data suggests that the pupil dilates earlier in Experiment 2 than in Experiment 1. To account for these differences the areas used to calculate pupil dilation and the size of these areas differ between the two experiments.
} 
ever, feed with a spoon. This occurred on average 2.7 times a day $(S D=1.2$, range $=0-5$ times $)$, feeding started on average 1.1 months prior to their visit $(S D=0.7$, range $=0$ 2.5 months). These experience measured did not correlate with the pupil dilation measures reported above.

Experiment 2 replicate and extend the pupil dilation findings of Experiment 1, demonstrating a larger pupil dilation to non-rational feeding actions than to actions that are equally novel (unfamiliar), but rational, given environmental constraints. This finding suggests that 6-month-old infants evaluate the function of observed actions, relating the trajectory of the observed action to the action goal and to the environment in which the action occurs. This retrospective evaluation of observed actions does not appear to be dependent on experience performing, or participating in, feeding (or eating) actions.

\section{General discussion}

The current study demonstrates a remarkable range of social competences in preverbal infants. Twelve-monthold infants rely on their own experience being feed to anticipate the goal of observed feeding actions. At the same time, infant's retrospective evaluations of rationality require less experience; both 6- and 12-month-old infants react with surprise if feeding actions are preformed in a non-rational manner. We suggest that anticipation of action goals is mediated by a direct matching process (Flanagan \& Johansson, 2003) whereas retrospective evaluations of rationality are dependent on more abstract well-formedness criteria as described by the teleological stance (Gergely \& Csibra, 2003).

\subsection{Anticipation requires experience}

Infants' anticipation of feeding actions demonstrates remarkable changes from 6 to 12 months of age. During the same time period infants gain a lot of experience being feed. Six-month-olds had been feed for about 1 month and they tracked the feeding actions in a reactive manner. Twelve-month-olds, on the other hand, had been feed for 7 months and they anticipate the action goal. This finding extends the results of Falck-Ytter et al. (2006) demonstrating that 12 -month-olds but not 6-month-olds anticipate manual actions that they have experience performing. In addition, the current study provides novel information about how infants anticipate everyday social interactions. We demonstrate that familiarity with an action is not sufficient to produce anticipatory gaze shifts. In fact, infants require ample experience (Fig. 4 ) with similar actions (Table 2) to anticipate action goals. This finding paints a rather different picture than the rapid experience dependency demonstrated by 3-month-old infants (Sommerville et al., 2005). It appears that only a small amount of experience is needed to alter infant's perception of goal directed actions (Sommerville et al., 2005) whereas infants require substantial experience to anticipate the goals of everyday social interactions.

This ability, to anticipate the goal of feeding actions, is not a simple binary process that separates those who poses action understand from those who do not. Instead, experience modulates the latency of goal directed gaze shifts in a graded manner. This phenomenon is illustrated in Fig. 4. When observing normal feeding actions 12-months-olds generally look at the recipient before the spoon arrives, however, their goal fixation latencies still correlate (.69) with days being feed. As such, infants with 240 days of experience being feed look at the recipient earlier than infants with 180 days of experience.

This is the first study to demonstrate such a clear connection between infants' everyday experience and their understanding of others actions. Similar effects have, however, been reported in adults. During passive viewing of chopstick manipulation Järveläinen, Schürmann, and Hari (2004) demonstrated a correlation (.83) between activity in the primary motor cortex and participant's chopsticks use in the last year. Järveläinen et al. argue that the close link between experience and underlying neural activity during action observation suggests the presence of a direct matching process mediated by the mirror neuron system. The fact that we rely on eye movements and not cortical activity makes a direct comparison between our results and those of Järveläinen et al. difficult. However, we suggest that the experience dependency observed in the current study results from a direct matching process, similar to that observed by Järveläinen et al. We argue that feeding experience strengthens motor representations; allowing precise timing between the arrival of the spoon and opening of the mouth during feeding, this timing is critical both when eating on ones own and when being feed by others. When observing others engage in feeding actions infants match their perception of these events with their own representation of similar interactions.

\subsection{Anticipations are organized around action goals}

Twelve-month-old infants' reactive gaze shifts are directed towards the recipients' head in the Rational condition and towards the hand in the Non-Rational condition; suggesting that infants attend to the actions performed in each of the two conditions. Despite of this, infants in both conditions look at the recipients face when anticipating the action goal. This tendency is persistent throughout the entire session and is present in both conditions. This suggests two things, that action anticipation is dependent on prior experience and that infants anticipatory gaze shifts are organized around action goals rather than perceived movements (Bekkering, Wohlschläger, \& Gattis, 2000; Gergely et al., 2002). In fact, even though the two conditions feature different action components both depict the same overall action goal; each action sequence ending with a piece of banana being placed in the recipients' mouth (successful feeding). It is this action goal that infants anticipate, regardless of how the action is performed.

This does not mean that the actual movements being observed are unimportant, quite the opposite. During the first feeding action of each movie infants performance is highly similar between conditions. Infants rely on prior experience being feed to anticipate that food will be delivered to the mouth; the average latency of this gaze shift being similar in both conditions. If presented with feeding 
actions that corresponds with their own experiences (Rational condition) 12-month-olds anticipations improve, decreasing the average goal fixation latency by $500 \mathrm{~ms}$ over the three feeding action of each video. We argue that the correspondence between the infants own experiences being feed and observed action components strengthens the direct matching process; resulting in fast goal fixations and a high correlation between experience and goal fixation latencies. If infants are presented with feeding actions that does not correspond with their own experience being feed, then the direct matching process is not strengthened; resulting in baseline performance throughout the entire session and a low correspondence between experience and goal fixation latencies.

\subsection{Retrospective evaluations of rationality requires less experience}

Numerous studies have demonstrated that young infants become surprised if self-propelled agents move on non-rational paths (Csibra, 2008; Csibra et al., 2003; Kamewari et al., 2005). We replicate and extend this effect in two experiments using a novel eye tracking paradigm that measure infants' pupil size in response to everyday action sequences; providing a unique demonstration of 6-monthold infants' ability to detect context specific violations of normal social interactions. As previously mentioned, 6months-olds experience being feed range from 0 to 3 months in Experiment 1 and from 0 to 5 months in Experiment 2, few of these infants had any experience eating on their own. Despite of this, both 6 and 12 month olds systematically dilated their pupil when observing non-rational feeding actions. Compared to the massive experience needed to anticipate the goal of feeding actions this suggests that less experience is needed to retrospectively evaluate rationality. Because we measure infants' pupil response on-line, as they observe repetitions of a single social interaction it is most likely that infants reactions are based on violations of expectations generated outside the lab environment. We argue that infants' evaluations of rationality are based on abstract well-formedness criteria, as described by the teleological stance (Csibra \& Gergely, 2007; Gergely \& Csibra, 2003). Infants assume that agents attempt to realize action goals in efficient manners (Rational condition of Experiment 1 and Rational and Unfamiliar condition of Experiment 2), and they become surprised when agents violate this principle (Non-Rational condition of Experiment 1 and Non-Rational and Unfamiliar condition of Experiment 2).

It is also important to emphasis that observed changes in pupil size are not caused by differences in luminance. The fact that the pupil dilates when luminance decreases (Winn, Whitaker, Elliott, \& Philips, 1994) makes it important to control for the amount of light reaching the eyes. In Experiment 1, the luminance from the back of the hand ( $\sim 200 \mathrm{fL}$ ) was brighter than the luminance from the mouth ( $\sim 150 \mathrm{fL})$ and hair ( $\sim 20 \mathrm{fL}$ ) of the recipient, suggesting pupil contraction when looking at the hand. Fig. 3 suggests the opposite relationship between fixation location and pupil size. Experiment 2 presents infants with identical luminance of the hand and mouth; however, the shadow created by the barrier darkened the hand as it moved under the barrier in the Rational and Unfamiliar condition relative to the Non-Rational and Unfamiliar condition. Despite of this, infants demonstrate the largest pupil dilation when observing the condition with the highest luminance (Non-Rational and Unfamiliar condition). The reverse effect would be expected if pupil dilation was caused by luminance differences.

\subsection{Everyday action understanding}

Most prior studies of action understanding in infancy has presented infant with fairly simple action sequences; including a small set of actions directed to a well defined goal (Falck-Ytter et al., 2006; Flanagan \& Johansson, 2003; Gergely, Nádasdy, Csibra, \& Bíró, 1995; Woodward, 1998). This tradition is in sharp contrast with the dynamic interaction used in the current study. We presented infants with two persons engaged in a sequence of different actions, including conversations, eating, and feeding. This means that infants are confronted with a broad range of potential actions and goals that change over time. We believe that the current results, as such, provide a more ecologically valid estimate of infant's action understanding during everyday social interactions. However, the added uncertainty that comes with multiple action sequences and diverse action goals (Gredebäck, Stasiewicz, Falck-Ytter, Rosander, \& von Hofsten, 2009; Rotman, Troje, Johnson, \& Flanagan, 2006) suggests that we should be careful about deducing minimum ages for anticipation and retrospective evaluation based on these results. The current study does not suggest a lower age limit for retrospective evaluations, since the youngest age group demonstrated this ability. We also find it likely that anticipatory gaze shifts will be observed in younger infants, if presented with a task that infants can master at an earlier age and with a simpler action sequence including a single actor and one well defined action goal.

In summary, we demonstrate that infants require less experience to evaluate rationality than what is needed to anticipate action goals. This finding should not change if both tasks are made easier. Based on these findings we argue for a dual process account of preverbal infant's action understanding. Infants simultaneously possess the ability to evaluate rationality based on well-formedness criteria (Gergely \& Csibra, 2003), and the ability to anticipate action goals based on a direct matching process (Falck-Ytter et al., 2006; Flanagan \& Johansson, 2003). Both of these abilities provide unique and important pieces of information that infants use when engaging in, and interpreting, the complex social interactions that define human interpersonal exchange.

\section{Acknowledgements}

This study was supported by Grants from the Norwegian Directorate for Children, Youth and Family Affairs (06/34707) to both authors. Correspondence to G. Gredebäck, the Cognitive Developmental Research Unit, Department of Psychology, University of Oslo, 0317 Blindern, Oslo, Norway. 


\section{References}

Bekkering, H., Wohlschläger, A., \& Gattis, M. (2000). Imitation of gestures in children is goal-directed. The Quarterly Journal of Experimental Psychology, 53A(1), 153-164.

Bradshaw, J. (1967). Pupil size as a measure of arousal during information processing. Nature, 216, 515-516.

Bruner, J. S. (1970). The growth and structure of skill. In K. Connolly (Ed.), Mechanisms of motor skill development (pp. 63-94). New York: Academic Press.

Carpenter, M., Akhtar, N., \& Tomasello, M. (1998). Fourteen- through 18month-old infants differentially imitate intentional and accidental actions. Infant Behavior and Development, 21(2), 315-330.

Csibra, G. (2008). Goal attribution to inanimate agents by 6.5 -month-old infants. Cognition, 107, 705-717.

Csibra, G., Bíró, S., Koós, O., \& Gergely, G. (2003). One-year-old infants use teleological representations of action productivity. Cognitive Science, 27, 111-133.

Csibra, G., \& Gergely, G. (2007). 'Obsessed with goals': Functions and mechanisms of teleological interpretation of actions in humans. Acta Psychologica, 124, 60-78.

Engel, K. C., Anderson, J. H., \& Soechting, J. F. (1999). Oculomotor tracking in two dimensions. Journal of Neurophysiology, 81(4), 1597-1602.

Eshuis, R., Coventry, K. R., \& Vulchanova, M. (2009). Predictive eye movements are driven by goals, not by the mirror neuron system. Psychological Science, 20(4), 438-440.

Falck-Ytter, T., Gredebäck, G., \& von Hofsten, C. (2006). Infants predict other people's action goals. Nature Neuroscience, 9(7), 878-879.

Flanagan, J. R., \& Johansson, R. S. (2003). Action plans used in action observation. Nature, 14(424), 769-771.

Gergely, G., Bekkering, H., \& Király, I. (2002). Rational imitation in preverbal infants. Nature, 415, 755.

Gergely, G., \& Csibra, G. (2003). Teleological reasoning in infancy: The naive theory of rational action. Trends in Cognitive Sciences, 7(7), 287-292.

Gergely, G., Nádasdy, Z., Csibra, G., \& Bíró, S. (1995). Taking the intentional stance at 12 months of age. Cognition, 56, 165-193.

Gredebäck, G., Johnsson, S., \& von Hofsten, C. (in press). Eye tracking in infancy research. Developmental Neuropsychology.

Gredebäck, G., Örnkloo, H., \& von Hofsten, C. (2006). The development of reactive saccade latencies. Experimental Brain Research, 173(1), 159-164.

Gredebäck, G., Stasiewicz, D., Falck-Ytter, T., Rosander, K., \& von Hofsten, C. (2009). Action type and goal type modulate goal directed gaze shifts in 14-month-old infants. Developmental Psychology, 45(4), 1190-1194.

Gredebäck, G., \& von Hofsten, C. (2007). Taking an action perspective on infant's object representations. In C. Von Hofsten \& K. Rosander (Eds.), From action to cognition (pp. 265-282). Amsterdam: Elsevier.
Iacoboni, M., \& Dapretto, M. (2006). The mirror neuron system and the consequences of its dysfunction. Nature Reviews Neuroscience, 7, 942-951.

Järveläinen, J., Schürmann, M., \& Hari, R. (2004). Activation of the human primary motor cortex during observation of tool use. Neurolmage, 23, 187-192.

Johnson, S. C., Shimitzu, Y. A., \& Ok, S. (2007). Actors and actions: The role of agent behavior in infants' attribution of goals. Cognitive Development, 22, 310-322.

Kamewari, K., Kato, M., Kanda, T., Ishiguro, H., \& Hiraki, K. (2005). Six-anda-half-month-old children positively attribute goals to human action and to humanoid-robot motion. Cognitive Development, 20, 303-320.

Király, I., Jovanovic, B., Prinz, W., Aschersleben, G., \& Gergely, G. (2003) The early origins of goal attribution in infancy. Consciousness and Cognition, 12, 752-769.

Land, M. F., \& Hayhoe, M. (2001). In what ways do eye movements contribute to everyday activities? Vision Research, 41, 3559-3565.

Libby, W. L., Lacey, B. C., \& Lacey, J. I. (1973). Pupillary and cardiac activity during visual attention. Psychophysiology, 10(3), 270-294.

Luo, Y., \& Baillargeon, R. (2005). Can a self-propelled box have a goal? Psychological Science, 16(8), 601-608.

Meltzoff, A. N. (1995). Understanding the intentions of others: Reenactment of intended acts by 18 -month-old children. Developmental Psychology, 31(5), 838-850.

Meltzoff, A. N. (2007). The 'like me' framework for recognizing and becoming an intentional agent. Acta Psychologica, 124, 26-43.

Rizzolatti, G., \& Craighero, L. (2004). The mirror-neuron system. Annual Review of Neuroscience, 27(16), 169-192.

Rotman, G., Troje, N. F., Johnson, R. M., \& Flanagan, J. R. (2006). Eye movements when observing predictable and unpredictable actions. Journal of Neurophysiology, 96, 1358-1369.

Shimizu, Y. A., \& Johnson, S. C. (2004). Infants' attribution of a goal to a morphologically unfamiliar agent. Developmental Science, 7(4), 425-430.

Sommerville, J. A., Woodward, A. L., \& Needham, A. (2005). Action experience alters 3-month-old infants' perception of others' actions. Cognition, 96, B1-B11.

Tryon, W. W. (1975). Pupillometry: A survey of sources of variation. Psychophysiology, 12(1), 90-93.

Võ, M. L. H., Jacobs, A. M., Kuchinke, L., Hofmann, M., Conrad, M., Schacht, A., et al. (2007). The coupling of emotion and cognition in the eye: Introducing the pupil old/new effect. Psychophysiology, 44.

Wagner, L., \& Carey, S. (2005). 12-month-old infants represent probable endings of motion events. Infancy, 7(1), 73-83.

Winn, B., Whitaker, D., Elliott, D. B., \& Philips, J. (1994). Factors affecting light-adapted pupil size in normal human subjects. Investigative Ophthalmology and Visual Science, 35(3), 1132-1137.

Woodward, A. L. (1998). Infants selective encode the goal of an actor's reach. Cognition, 69(1), 1-34. 